B. Bielekova, MD

N. Richert, MD, PhD

M.L. Herman

J. Ohayon, CRNP

T.A. Waldmann, MD

H. McFarland, MD

R. Martin, MD

G. Blevins, MD

Address correspondence and reprint requests to Dr. Bibiana Bielekova, Neuroimmunology Branch (NIB), National Institute of Neurological Disorders and Stroke, NIH, Building 10, Room 5C103, Bethesda, MD 20892 Bibi.Bielekova@nih.gov
Supplemental data at www.neurology.org

\title{
Intrathecal effects of daclizumab treatment of multiple sclerosis
}

全

\section{ABSTRACT}

Objectives: We previously reported that daclizumab, a humanized monoclonal antibody against CD25, reduced contrast-enhancing lesions (CEL) in patients with multiple sclerosis (MS) who were suboptimal responders to interferon- $\beta$ and that this response correlated with expansion of CD56 bright NK cells. These data have been reproduced in a placebo-controlled multicenter trial (CHOICE study). The current study investigates whether daclizumab monotherapy reduces CEL in untreated patients with relapsing-remitting MS (RRMS) and the effects of daclizumab on the intrathecal immune system.

Methods: Sixteen patients with RRMS with high inflammatory activity were enrolled in an open-label, baseline-vs-treatment, phase II trial of daclizumab monotherapy for 54 weeks and followed by serial clinical and MRI examinations and immunologic biomarkers measured in the whole blood and CSF.

Results: The trial achieved predefined outcomes. There was an $87.7 \%$ reduction in brain CEL (primary) and improvements in Multiple Sclerosis Functional Composite (secondary), Scripps Neurologic Rating Scale, and Expanded Disability Status Scale (tertiary) outcomes. There was significant expansion of CD56 bright NK cells in peripheral blood and CSF, with resultant decrease in T cells/NK cells and B cells/NK cells ratios and IL-12p40 in the CSF. Surprisingly, CD25 Tac epitope was equally blocked on the immune cells in the CSF and in peripheral blood.

Conclusions: Daclizumab monotherapy inhibits formation of MS plaques in patients with RRMS and immunoregulatory NK cells may suppress activation of pathogenic immune responses directly in the CNS compartment.

Classification of evidence: The study provides Class III evidence that daclizumab reduces the number of contrast-enhancing lesions in treatment-naive patients with RRMS over a 54-week period. Neurology ${ }^{\circledR} 2011 ; 77: 1877-1886$

\section{GLOSSARY}

$\mathbf{A b}=$ antibodies; $\mathbf{B F V}=$ brain fractional volume; $\mathbf{C E L}=$ contrast-enhancing lesion; $\mathbf{E D S S}=$ Expanded Disability Status Scale; FLAIR = fluid-attenuation inversion recovery; FSE = fast spin echo; IFN- $\boldsymbol{\beta}=$ interferon- $\beta$; IL $=$ interleukin; $\mathbf{m A b}=$ monoclonal antibody; $\mathbf{M O A}=$ mechanism of action; $\mathbf{M F I}=$ mean fluorescence intensity; $\mathbf{M S}=$ multiple sclerosis; MSFC = Multiple Sclerosis Functional Composite; MTR = magnetization transfer ratio; NRS = Neurological Rating Scale; RRMS = relapsing-remitting multiple sclerosis; T2LV = T2 lesion volume; TNF $\boldsymbol{\alpha}=$ tumor necrosis factor $\alpha$.

We previously reported that patients with persistent MS disease activity on interferon- $\beta$ (IFN- $\beta$ ) therapy were successfully treated by the addition of daclizumab, a humanized monoclonal antibody (mAb) against the $\alpha$-chain of the interleukin (IL)-2 receptor (CD25). ${ }^{1-3}$ The efficacy of therapeutic response correlated with the expansion of immunoregulatory CD $56^{\text {bright }}$ NK cells, ${ }^{4}$ suggesting that this measurement may become a useful biomarker in therapeutic development of daclizumab. ${ }^{2}$ All of these observations have now been reproduced in an independent, multicenter, placebo-controlled clinical trial of daclizumab in MS (CHOICE study). ${ }^{5}$

Our subsequent studies demonstrated that CD $56^{\text {bright }}$ NK cells can inhibit adaptive immune responses by killing autologous activated $\mathrm{T}$ cells, ${ }^{4}$ but it remained uncertain whether

\footnotetext{
From the Neuroimmunology Branch, National Institute of Neurological Disorders and Stroke (B.B., N.R., M.L.H., J.O., H.M., R.M., G.B.), and Metabolism Branch, National Cancer Institute (T.A.W.), National Institutes of Health, Bethesda, MD; Department of Neuroimmunology (R.M.), Zurich University Hospital, Zurich, Switzerland; and Division of Neurology (G.B.), University of Alberta, Edmonton, Canada.

Study funding: Supported by the Intramural Research Program of the NIH/NINDS. Daclizumab was provided free of charge by Roche, under collaborative agreement with T.A.W., NCI.

Disclosure: Author disclosures are provided at the end of the article.
} 
CD56 $6^{\text {bright }}$ NK cells can enter the CNS. Studies in experimental autoimmune encephalomyelitis suggested that NK cells need to access the intrathecal compartment in order to exert full protective effect against destructive inflammation of the CNS. ${ }^{6}$ Furthermore, CD $56^{\text {bright }} \mathrm{NK}$ cells are also expanded and activated by IFN- $\beta$ treatment alone, ${ }^{2,7}$ raising the question as to whether monotherapy with daclizumab would be as effective as combination therapy with IFN- $\beta$.

Therefore, in the current study we sought to answer 3 important questions about daclizumab therapy in MS: 1) What is the therapeutic efficacy of daclizumab monotherapy in patients with RRMS? 2) Do expanded CD $56^{\text {bright }} \mathrm{NK}$ cells reach the intrathecal compartment? And if so; 3) How does their transmigration into the CNS affect other lymphocyte subpopulations and markers of MSrelated inflammation?

METHODS Trial design, standard protocol approvals, registrations, and patient consents. This was a singlecenter, open-label, baseline-vs-treatment crossover phase II trial (figure 1; clinical trials.gov identifier NCT00071838) approved by the institutional review board of National Institute of Neurological Disorders and Stroke. Written informed consent was obtained from all study subjects.

Sample size calculation, determined from analysis of our natural history cohort monthly MRI studies, required 15 patients to finish dosing in order to detect a $50 \%$ reduction in CEL from 4 baseline MRIs to 4 treatment MRIs with a power of 0.8 and an $\alpha$ of 0.05 (2-sided).

The study population consisted of 16 patients with RRMS (table e-1 on the Neurology ${ }^{\circledR}$ Web site at www.neurology.org) diagnosed by McDonald criteria ${ }^{8}$; ages 18-65 years, Expanded Disability Status Scale (EDSS ${ }^{9}$ range $1.0-5.5$, with a mean CEL $\geq 0.5$ lesions per month during the baseline period of 12 weeks. All eligible patients received IV daclizumab $(1 \mathrm{mg} / \mathrm{kg})$ at week 0 and 2 , then every 4 weeks up to week 54 . Patients were followed by monthly clinical and brain MRI examinations. Patientspecific average values of quantitative clinical and MRI parameters, collected during 12 weeks of baseline, were compared with average of 4 monthly measurements collected during primary treatment phase (weeks 18-30) and completion of treatment phase (weeks 42-54) as indicated in figure 1A.

MS relapses were defined by Schumacher' ${ }^{10}$ criteria and were treated by IV methylprednisolone ( $1 \mathrm{~g} /$ day for 5 days). MRI and clinical data within 28 days of methylprednisolone therapy were replaced by data from the following month, leading to proportionate expansion of baseline or treatment periods.

The primary outcome was reduction of new CEL from baseline to primary treatment phase. Secondary outcome measures were change in Multiple Sclerosis Functional Composite $\left(\mathrm{MSFC}^{11}\right)$ and reduction of new CEL from baseline to completion of treatment phase. Tertiary outcome measures were as follows: change in mean values of EDSS, Scripps Neurological
Rating Scale (Scripps NRS ${ }^{12}$ ), volume of CEL, T2 lesion volume (T2LV), whole brain magnetization transfer ratio (MTR), brain fractional volume (BFV), and immunologic parameters between baseline and treatment phases.

MRI. Contiguous 3-mm axial images at $1.5 \mathrm{~T}$ used dual echo fast spin echo (FSE) T2-weighted and fluid-attenuation inversion recovery (FLAIR) images. ${ }^{13}$ MTR imaging used T1weighted images before and after an Rf saturation pulse $(600 \mathrm{~Hz}$ below water frequency)..$^{14}$ Contrast-enhanced $\mathrm{T} 1 \mathrm{~W}$ images were obtained 5 minutes post injection of Magnevist $(0.1 \mathrm{mmol} / \mathrm{kg}$; Bayer Healthcare Pharmaceuticals).

CEL were recorded on hard copy films by a radiologist who was not involved in the clinical care of the patients (N.R.) and images were transferred to a UNIX Workstation (Sun Microsystems). All image sequences from sequential MRI examinations were coregistered to the initial T1-weighted image obtained at the entry examination $\left(\mathrm{t}=-12\right.$ weeks). ${ }^{15} \mathrm{~A}$ skull-stripped mask image from the entry $\mathrm{T} 1$-weighted scan was sequentially applied to each MRI sequence from all subsequent examinations to ensure analysis of the same volume of brain parenchyma. ${ }^{13} \mathrm{~T} 2 \mathrm{LV}$ was calculated from registered FLAIR images using the semiautomated thresholding technique (PVWAVE). ${ }^{1}$ CEL volumes were determined using the semiautomated MEDx program. ${ }^{1}$ MTR images were analyzed as previously described..$^{14}$ Brain atrophy was quantified using ADPK mean to determine brain fractional volume $(\mathrm{BFV}) .^{13}$

Flow cytometry of whole blood and CSF samples. Peripheral blood was collected into anticoagulated (EDTA) tubes and processed within 2 hours of collection as described. ${ }^{4}$ Paired CSF samples $(15-20 \mathrm{~mL})$ were transported on ice and spun $(250$ $g, 10$ minutes, $4^{\circ} \mathrm{C}$ ) within 10 minutes of collection. The CSF cell pellet was resuspended in $700 \mu \mathrm{L}$ of ice-cold FACS buffer (phosphate-buffered saline, $1 \%$ fetal calf serum, $0.1 \% \mathrm{Na}$ azide) and aliquoted into a 96-well plate at $100 \mu \mathrm{L} /$ well for quadruple labeling with saturating concentrations of conjugated antibodies (Ab). After osmotic red blood cell lysis, ${ }^{16}$ blood samples were aliquoted to identical 96-well plate and concentrated cell pellets were stained in parallel with identical $\mathrm{Ab}$ as CSF cells for 30 minutes on ice. After washing, cells were acquired immediately by flow cytometry (FACS Calibur) and analyzed with FACS Diva software (both BD Biosciences). Lymphocytes and monocytes were gated based on forward and side scatter and subgated based on isotype controls. The number of acquired live lymphocytes ranged between 15,000 and 20,000 events/blood sample and 1,000 and 10,000 events/CSF sample. For CSF subpopulations with low cell numbers (e.g., CD $56^{\text {bright }} \mathrm{NK}$ cells), data were measured in at least 2 different staining tubes and results were considered significant only when both staining tubes provided consistent results.

Cytometric bead array and ELISA. CSF supernatants were concentrated (up to 10-fold) using Millipore Amicon Ultra 3 $\mathrm{kDa}$ filters and analyzed in multiplex for IL-6, IL-7, IL-8, IL-10, IL-17, IL-12p40 and p70, IL-21, IFN- $\gamma$, oncostatin M, tumor necrosis factor $\alpha$ (TNF $\alpha)$, LT- $\alpha$, vascular endothelial growth factor, CX3CL1, and granzyme B using BD cytometric bead array (CBA Flex Sets) and IL-23 was measured by R\&D Quantikine ELISA assay according to the manufacturer's instructions. Detection limits and CSF dilutions are outlined in table e-2. Data were acquired on an LSR II flow cytometer and analyzed using FCAP array software (all BD Biosciences).

Statistical analyses. For primary and secondary outcomes, for each patient, period average was computed by averaging 4 monthly 
Figure 1 Trial design and flow diagram

A

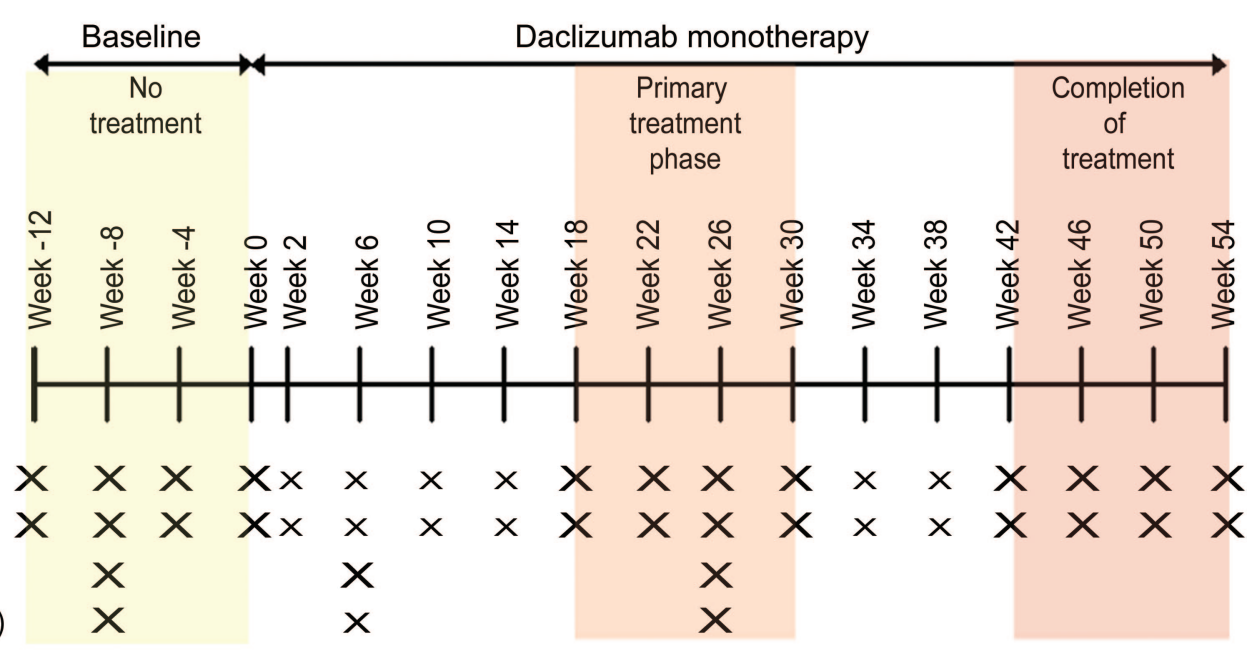

B

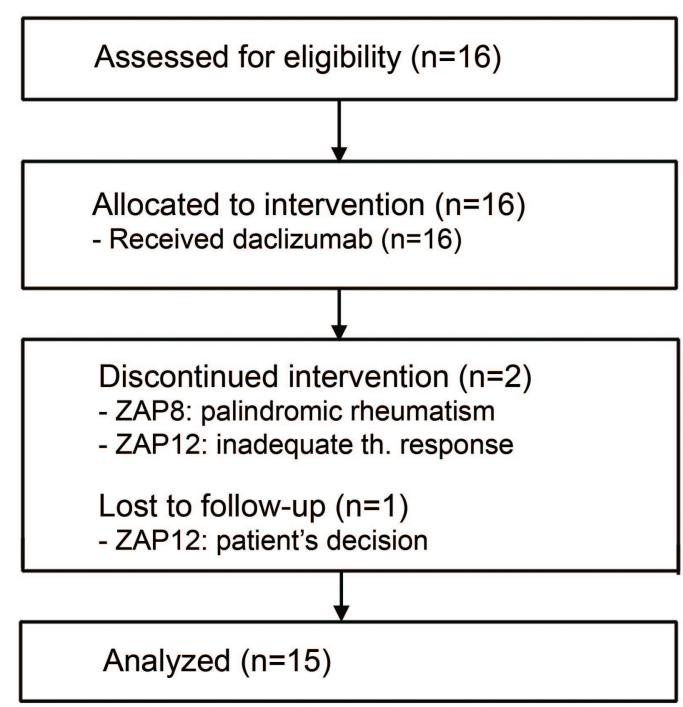

(A) Trial design: patients had 4 monthly clinical and MRI examinations during untreated "baseline" before daclizumab dosing was initiated at $1 \mathrm{mg} / \mathrm{kg}$ IV. The first 2 doses were 2 weeks apart, after which patients received daclizumab infusion every 4 weeks for total of 54 weeks. Although clinical and MRI examinations were performed monthly, only 3 predefined 12-week periods were considered in data analysis: the baseline period (week -12 through week 0), primary treatment phase (weeks 18-30), and completion of treatment phase (weeks 42-54). For all outcomes, patient-specific averages of 4 MRI/ clinical examinations were computed for all 3 phases and analyzed as described in Methods. For immunologic outcomes, matched CSF and blood samples were collected once during pretreatment baseline and once after week 26 of treatment. There was optional CSF collection at patient's discretion at week 6 of daclizumab treatment. (B) CONSORT statement 2010 flow diagram.

values for baseline, primary treatment, and completion of treatment periods. Wilcoxon signed-rank test was used for the analysis of primary and secondary outcome measures. Repeated-measures analysis of variance on ranks or when permitted (passing normality and equal variance tests) one-way repeated-measures analysis of variance was used for the analysis of tertiary outcome measures. Tukey test was used for all pairwise comparisons. To account for multiple comparisons, a $p$ value of 0.01 was used as the cutoff for statistical significance. All effort was taken to populate missing data through unscheduled visits (e.g., repeated MRI scans). In rare instances (e.g., technical problem with CSF flow cytometry), the missing data could not be repopulated and they did not contribute to the analysis.

RESULTS Safety and tolerability. A total of 14 out of 16 patients $(87.5 \%)$ finished 54 weeks of daclizumab dosing (table e-1 and figure 1B). In 2 patients (12.5\%), daclizumab was stopped by the investigators; patient ZAP8 developed palindromic rheumatism after 4 doses of daclizumab, which responded to drug withdrawal. This patient was followed for the full 54 weeks and the data are included in intention-to-treat analysis. Patient ZAP12 had unusually high MS disease activity during pretreatment baseline (27 CEL at the initiation of daclizumab dosing) and the disease activity did not respond to total of 3 doses of daclizumab; therefore the patient was withdrawn from the study to undergo more aggressive therapeutic management and was lost to follow-up after week 18. This patient was replaced (by patient 


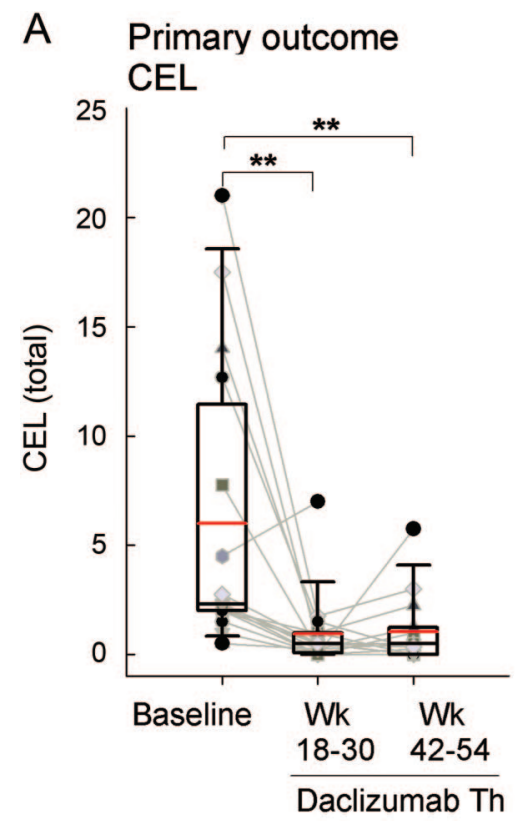

\section{B Secondary outcome MSFC}

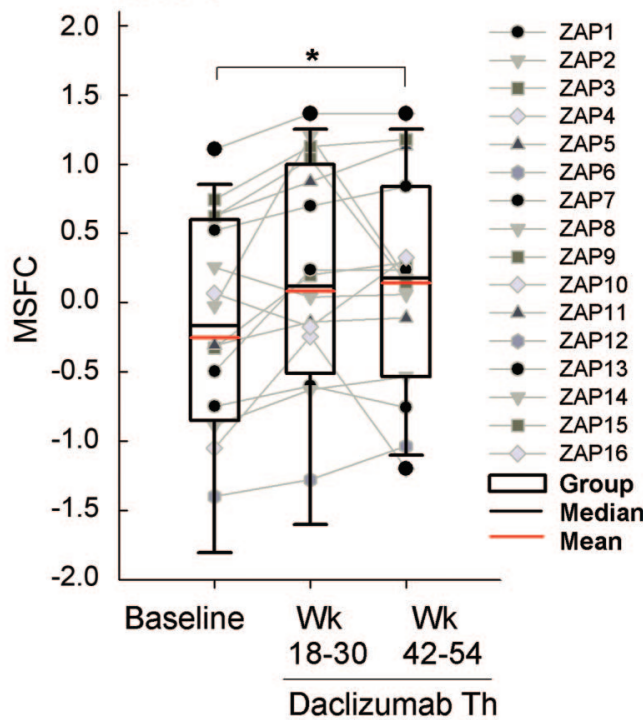

\section{C \\ Tertiary outcome Scripps NRS}

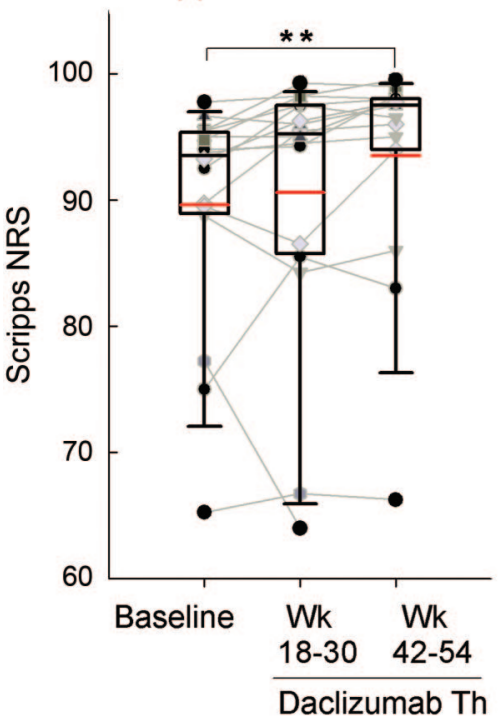

\section{D}
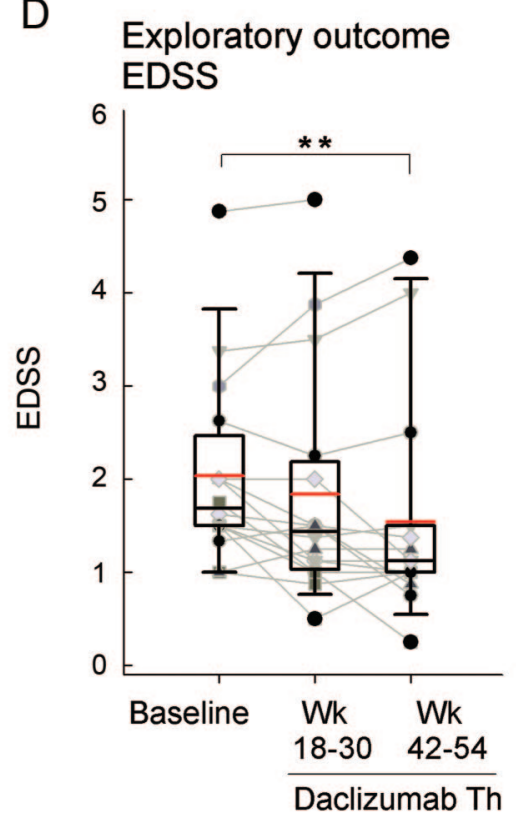

(A-D) Period averages were computed for each patient as described in Methods and are depicted as dot and line plots. Group data are depicted as open box blots, with group medians represented by black and means by red horizontal lines. Statistically significant differences are highlighted by asterisk (*p $\leq 0.01$ and $\left.{ }^{* *} p \leq 0.001\right)$. CEL $=$ contrastenhancing lesion; EDSS = Expanded Disability Status Scale; MSFC = Multiple Sclerosis Functional Composite; NRS = Neurological Rating Scale.

ZAP16) to obtain 15 patients who contributed to the data analysis.

Overall, daclizumab therapy was well tolerated and side effects were mild to moderate (table e-1). As in previous studies, ${ }^{1}$ we observed nonsignificant increase in infection rate (from 0.54 per patient-year to 0.87 per patient-year, mostly upper respiratory and urinary tract); transient changes in laboratory results, which did not require dose adjustments; and other adverse events included palindromic rheumatism, dermatographia, sebopsoriasis, and headaches.

Imaging and clinical outcomes. The average number of new CEL, the primary outcome measure, was reduced from a median of 2.042 at baseline to 0.250 at primary treatment phase $(87.6 \%$ inhibition, $p<$ 0.001; Wilcoxon signed-rank test). Similar reduction was observed for total CEL (figure 2A) and for volume 
of CEL (from $0.328 \mathrm{~mm}^{2}$ to $0.034 \mathrm{~mm}^{2}$; $89.7 \%$ inhibition, $p<0.001)$. This inhibition of CEL was sustained until the end of treatment (figure 2A).

For secondary outcomes, we observed group improvement for MSFC from -0.165 to 0.175 ( $p=$ 0.015; figure 2B) and for Scripps NRS from 93.5 to 97.5 ( $p=0.001$; figure $2 \mathrm{C}$ ). We found reduction in EDSS, from 1.688 to 1.125 ( $p=0.001$; figure $2 \mathrm{D}$ ).

We observed no significant change in the T2 LV or in whole brain MTR. BFV decreased from 0.8740 at baseline to 0.8460 at the completion of treatment $(p=0.004)$. The individualized changes in BFV correlated with the individualized changes in the volume of CEL $\left(\mathrm{R}_{\text {Spearman }}=0.643\right.$; $p=0.00934)$. Relapse rate decreased from 0.54 per patient-year to 0.12 per patient-year $(77.8 \%$ inhibition, nonsignificant).

Immunologic outcomes. Daclizumab treatment had no effect on CSF lymphocyte counts. Similarly, daclizumab therapy had no effect on CD4/CD8 T cell or $\mathrm{T}$ cell/B cell ratios in blood or CSF, even though CD4 $\mathrm{T}$ cells were significantly enriched (figure $3 \mathrm{~A}$ ) and $\mathrm{B}$ cells were significantly diminished (figure $3 \mathrm{~B}$ ) in the CSF in comparison to blood. However, we observed an increase in the proportion of NK cells in the blood and CSF during daclizumab treatment, resulting in significant decreases in CD4/NK (figure 3C) and CD8/NK cell ratios (figure $3 \mathrm{D}$ ). The $\mathrm{B}$ cell/ $\mathrm{NK}$ ratio was reduced only in the CSF (figure $3 \mathrm{E}$ ).

The CD56 mean fluorescence intensity (MFI) on NK cells also increased during daclizumab treatment (figure $3 \mathrm{~F}$ ), consistent with the daclizumab-driven expansion of CD $56^{\text {bright }} \mathrm{NK}$ cells. However, we encountered a problem with gating of $\mathrm{CD} 56^{\text {bright }} \mathrm{NK}$ cells. While in peripheral blood, we could identify these cells based on their high expression of both CD56 and CD44 compared to CD56 ${ }^{\mathrm{dim}}$ counterparts; in CSF all NK cells expressed very high levels of CD44 (figures 3D and 4). In order to quantify CD $56^{\text {bright }} \mathrm{NK}$ cells, we set the gate on peripheral blood sample and used an identical gate for CSF lymphocytes (figure 4). Using this strategy, we observed expansion of CD56 $6^{\text {bright }} \mathrm{NK}$ cells both in peripheral blood and in the CSF (figure $3 \mathrm{H}$ ).

Next, we assessed expression of IL-2R chains: $\mathrm{CD} 25$, the target of daclizumab, and CD122, the IL-2R- $\beta$ chain (figure 5 ). We observed that daclizumab bound strongly to $\mathrm{T}$ cells (CD4 $+\mathrm{T}$ cells depicted, figure 5A) and NK cells (figure 5B), resulting in complete blockade of CD25-Tac epitope (which is required for IL-2 binding) in both peripheral blood and CSF. In contrast, 7G7 epitope, which is outside of daclizumab/IL-2 binding domain, was decreased only on T cells, both in blood and CSF (figure 5C), but not on NK cells (figure 5D). The residual staining of CD25-7G7 Ab during daclizumab therapy indicated that some CD25 molecules remained expressed on the surface of both T cells and NK cells. Interestingly, CSF NK cells expressed considerably higher levels of CD25 before daclizumab treatment compared to blood NK cells.

We observed no changes in CD122 expression induced by daclizumab on $\mathrm{T}$ cells, although CSF $\mathrm{T}$ cells had higher expression of CD122 in comparison to blood $\mathrm{T}$ cells (figure $5 \mathrm{E}$ ). In contrast, CD122 MFI was mildly enhanced on blood NK cells, consistent with expansion of CD $56^{\text {bright }} \mathrm{NK}$ cells that express higher levels of CD122 compared to CD56 ${ }^{\text {dim }} \mathrm{NK}$ cells.

We also evaluated expression of chemokine receptors CCR5 and CXCR3. Consistent with previous reports, we observed that almost all $\mathrm{T}$ cells and $\mathrm{NK}$ cells in the CSF expressed CXCR3, but daclizumab induced no changes to CXCR3 or CCR5 expression on either T cells or NK cells (figure 4; only T cells are displayed). When evaluating signaling chains of other cytokines that compete with IL-2 for common $\gamma$-chain (i.e., IL-4R $\alpha$ and IL-7R $\alpha$ ), we observed a nonsignificant trend for their increased expression on $\mathrm{T}$ cells during daclizumab therapy $(p=0.048$; data not shown).

Daclizumab-induced changes in intrathecal production of soluble inflammatory mediators. Using a highly sensitive CBA assay on up to 10-fold concentrated CSF, we were still able to consistently detect only $3 / 16$ soluble mediators (table e- 2 ). The levels of IL-6 and IL- 8 were not changed, but IL-12p40 levels decreased by $61.1 \%(p=0.006)$ during daclizumab therapy. IL-12p40 is shared subunit of both IL-12 and IL-23. Unfortunately, both IL-12 and IL-23 measurements were below detection limit of our assays.

DISCUSSION This open-label phase II trial using objective neuroimaging measure of formation of focal MS lesions demonstrates efficacy of daclizumab monotherapy as first-line therapy of RRMS. The observed level of inhibition of CEL as well as improvement of clinical parameters are comparable to previous studies of daclizumab combination therapy with IFN- $\beta$ in MS. ${ }^{1-3,5,17}$ Although we interpret improvement in clinical scales cautiously due to openlabel trial design, the improvement in MSFC and continuous improvement in EDSS from weeks $18-30$ to weeks $42-54$ imply that these changes are unlikely due to reversal of relapse-related disability. Rather these data suggest that the suppression of brain inflammation may allow endogenous repair mechanisms to partially restore function in early 
A

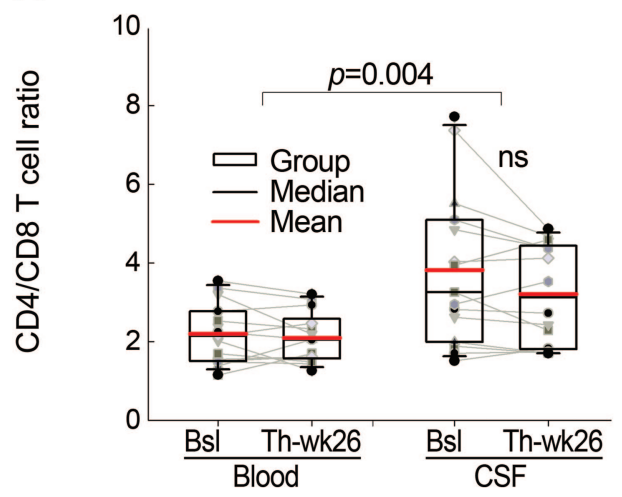

C

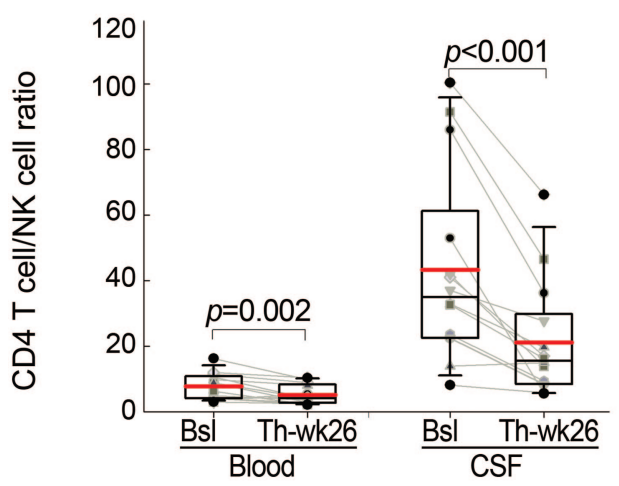

$E$

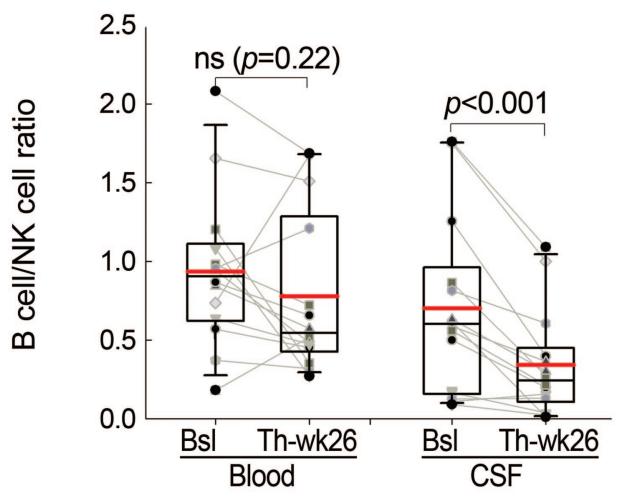

G

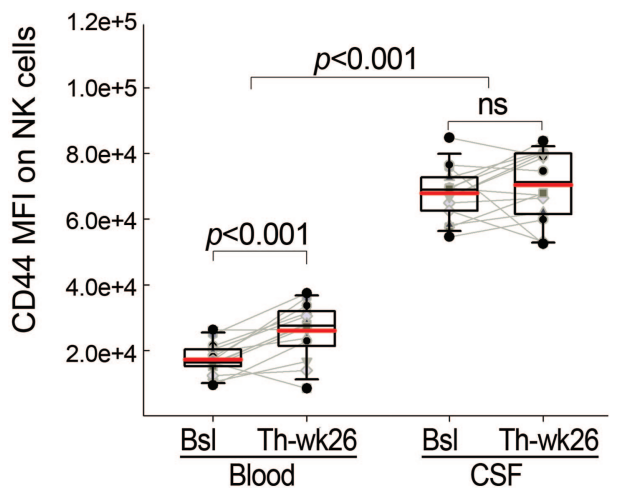

B

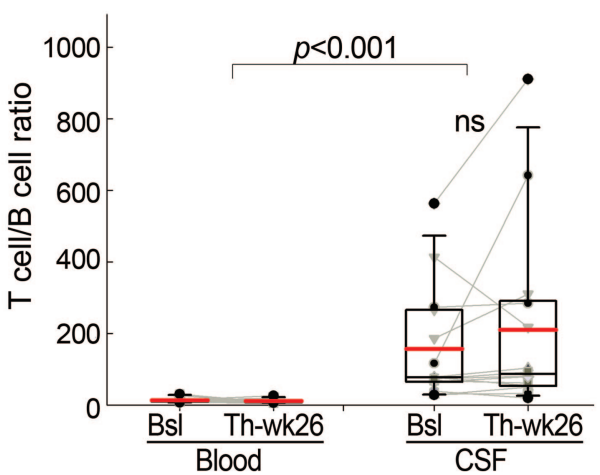

D

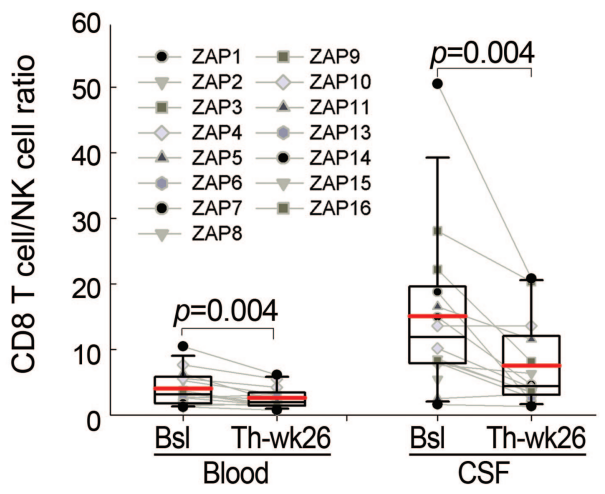

F

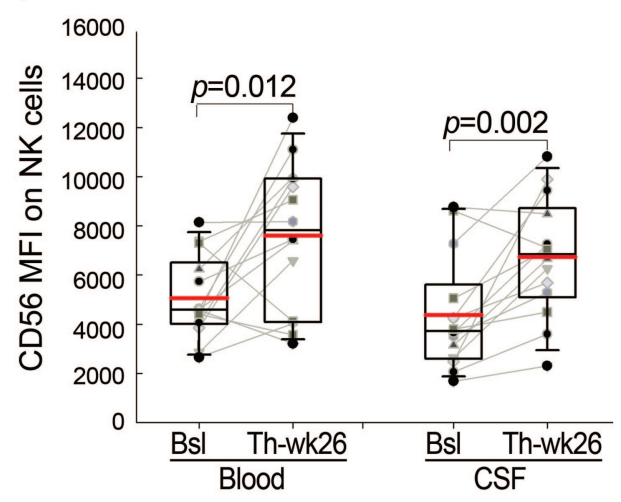

$\mathrm{H}$

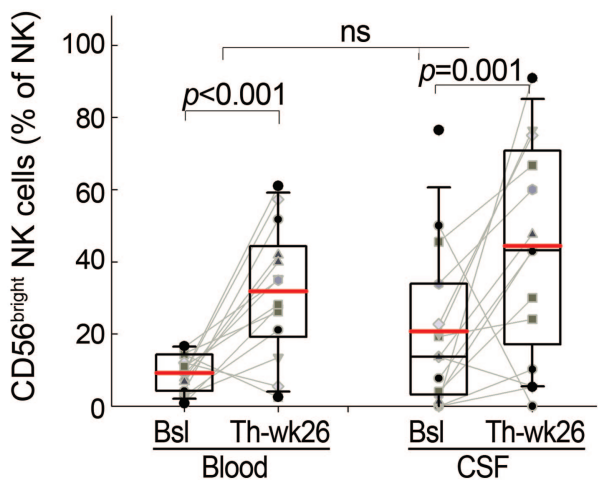

(A-H) Patient-specific data are depicted as dot and line blots. Group data are depicted as open box blots, with group medians represented by black and means by red horizontal lines. Statistically significant differences are highlighted with exact $p$ values depicted in the figure. MFI = mean fluorescent intensity. 
Baseline

Blood

CD4+

$T$ cells
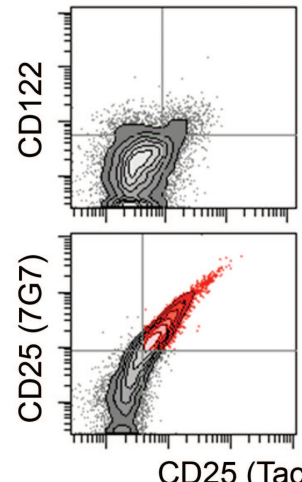

CD25 (Tac epitope)

T cells

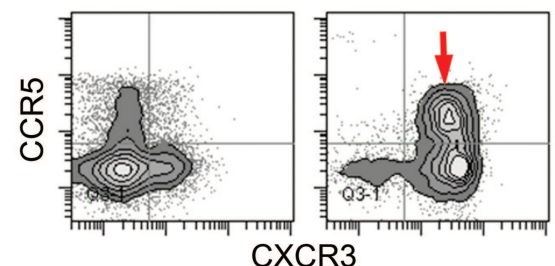

NK cells

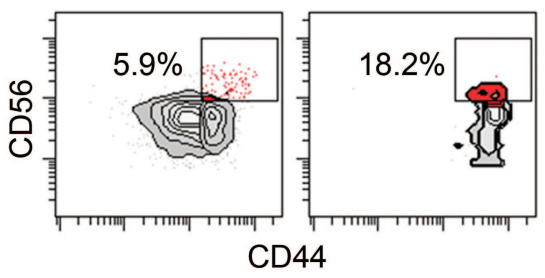

Dac Th wk 26

Blood
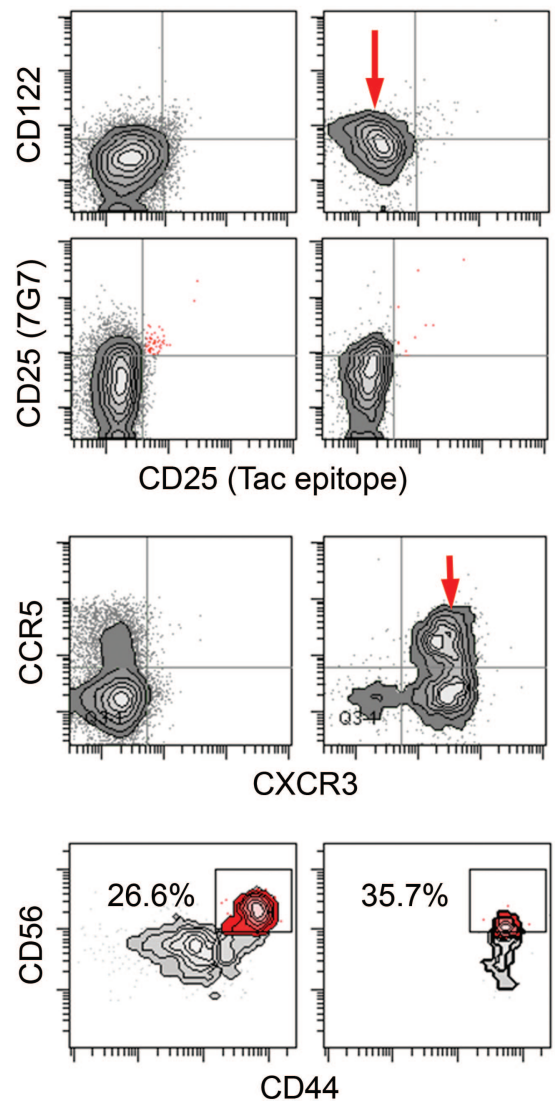

Flow cytometry staining on immune cells (CD4 + T cells: first 2 rows, CD3 + T cells: third row, and CD3-/CD56 + NK cells: fourth row) in the blood (left panels) and paired CSF samples (right panels) isolated during pretreatment baseline and after week 26 of daclizumab therapy in a representative patient. The red arrow in the first row highlights higher expression of CD122 (interleukin [IL]-2R- $\beta$ chain) in the CSF as compared to blood. In the second row, T cells positive for CD25 (both Tac and 7 G7 epitopes) are highlighted in red. In the third row, the red arrow highlights significantly higher expression of CXCR3 and CCR5 chemokine receptors on the T cells in the CSF as compared to blood, but there is no change with daclizumab therapy. Quadrant gates were set based on appropriate isotype controls for each staining combination. In the fourth row, CD56 bright NK cells (highlighted in red) are gated in the blood based on their higher expression of CD56 and CD44 in comparison to CD56 dim NK cell counterparts. However, in the CSF, all NK cells express uniformly high CD44. The proportion of NK cells that were gated as CD56 $6^{\text {bright }}$ both in the blood and CSF are depicted as number in each contour blot.

stage MS. The significant decline in BFV may be due to preprogrammed neurodegeneration, but more likely represents pseudoatrophy, because of its strong correlation with the change in the volume of CEL.

Evaluation of the immune cells in the CSF demonstrated that daclizumab has very different mechanism of action (MOA) compared to other $\mathrm{mAb}$ therapies used in MS, specifically natalizumab, rituximab, and possibly also alemtuzumab. While other $\mathrm{mAb}$ decrease immune cells in the intrathecal compartment by decreasing migration ${ }^{18,19}$ or by depletion, ${ }^{20}$ daclizumab exerts true immunomodulatory effects. One drawback of our study is that quantification of CSF leukocytes was performed on unspun CSF by NIH clinical laboratory, which is not as sensitive as quantification of leukocytes after concentrat- ing CSF by centrifugation. ${ }^{20}$ Nevertheless, the fact that daclizumab is a nondepleting $\mathrm{mAb},{ }^{4}$ that there was no effect of daclizumab on chemokine receptors or adhesion molecules, and that daclizumab did not change CD4/CD8 $\mathrm{T}$ cell ratios or $\mathrm{T}$ cell/B cell ratios in the CSF all support our conclusion that daclizumab does not limit access of immune cells to the intrathecal compartment. Instead, we observed significant enhancement of NK cell numbers, specifically immunoregulatory $\mathrm{CD} 56^{\text {bright }} \mathrm{NK}$ cells, in peripheral blood and CSF. This is reassuring, because it implies that $\mathrm{CD} 56^{\text {bright }} \mathrm{NK}$ cells can regulate (i.e., kill) activated pathogenic T cells directly in MS tissue. $^{6}$

We were surprised to find that the CD25 Tac epitope was completely blocked in the CSF and 
A
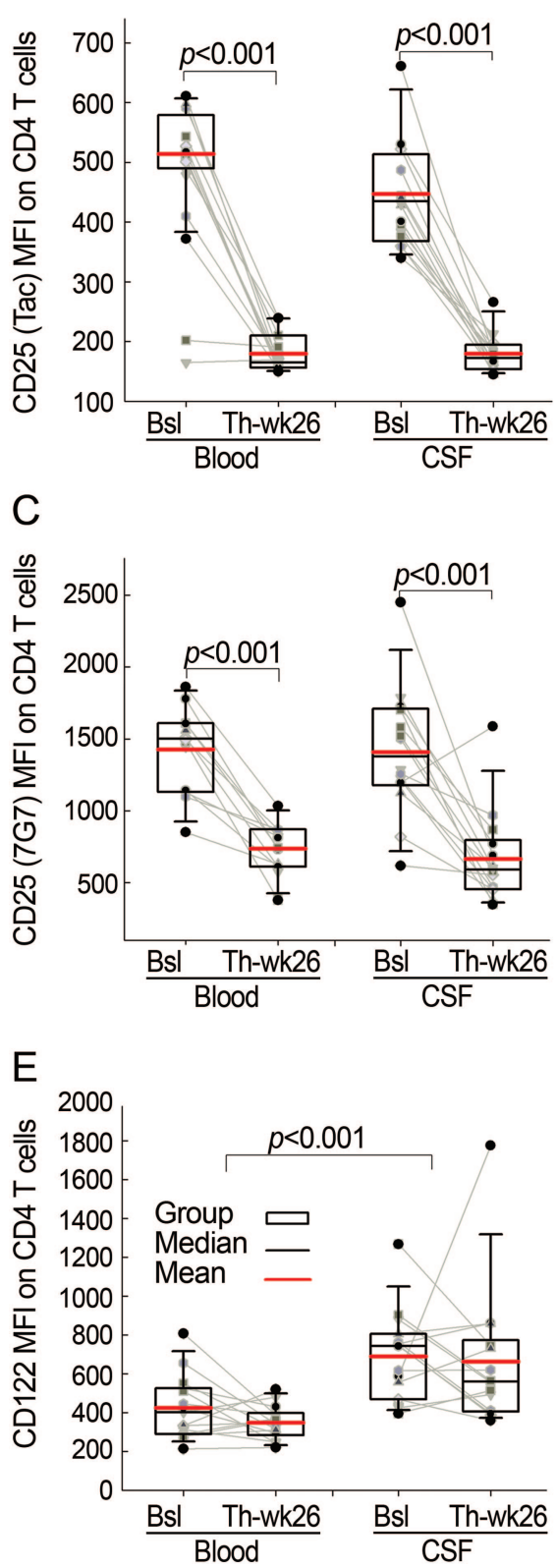

B

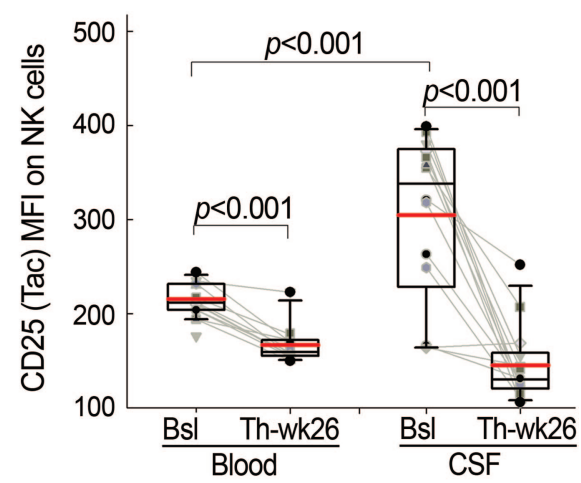

D

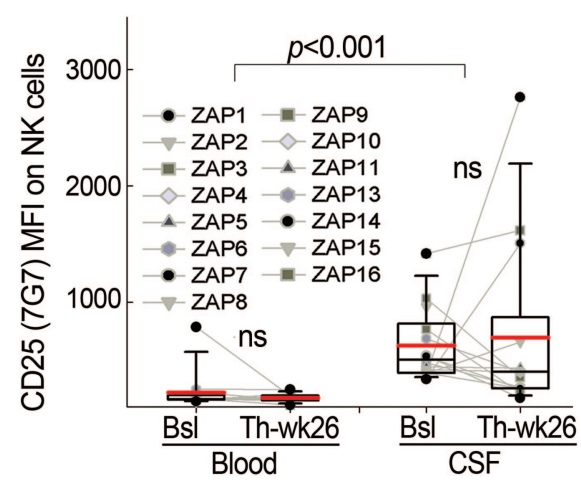

$\mathrm{F}$

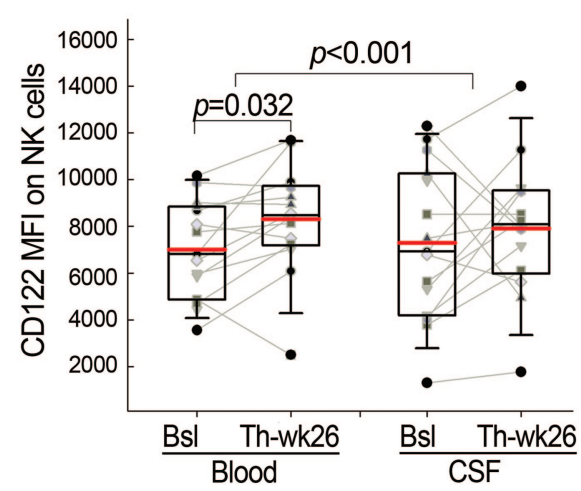

(A-F) Patient-specific data are depicted as dot and line blots. Group data are depicted as open box blots, with group medians represented by black and means by red horizontal lines. Statistically significant differences are highlighted with exact $p$ values depicted in the figure. MFI = mean fluorescent intensity.

blood during daclizumab treatment. Although we cannot measure daclizumab in the CSF, we estimate that only $0.1 \%$ of serum daclizumab gains access to the intrathecal compartment based on studies with other mAbs. ${ }^{21}$ Such low (i.e., $10 \mathrm{ng} / \mathrm{mL}$ ) intrathecal concentrations of daclizumab would be insufficient to block de novo synthesis of CD25 induced by T-cell activation within the CNS. Therefore, our results suggest that daclizumab saturated CD25 Tac epitope in the periphery, before these lymphocytes migrated into the intrathecal compartment, and $\mathrm{T}$ cells were either not reactivated in the CNS, or, alter- natively, those that were activated were effectively killed by $\mathrm{CD} 56^{\text {bright }} \mathrm{NK}$ cells and did not gain access to the CSF during daclizumab treatment.

Unfortunately, it is impossible to directly dissect these alternative explanations with current methodologies in humans. We attempted to answer these questions by measuring intrathecally produced cytokines, indirectly reflecting immune activation in MS lesions. Despite employing methodology that is at least 10-fold more sensitive than ELISA and despite our effort to further concentrate CSF, we failed to detect any of the T-cell-secreting cytokines that are 
postulated to play a pathogenic role in MS. Only IL-6, IL-8, and IL-12p40 were detected in our assays. Both IL- 6 and IL- 8 are produced by many different cell types, including nonimmune cells (e.g., fibroblasts, endothelial cells, and astrocytes), and the CSF levels of these cytokines were not affected by daclizumab treatment. Conversely, IL-12p40 is produced exclusively by immune cells, e.g., macrophages and dendritic cells, microglia, activated $\mathrm{B}$ cells, and, to a lesser extent, by $\mathrm{T}$ cells. We detected robust decrease in IL-12p40 CSF levels during daclizumab treatment, although neither IL-12 nor IL-23 was detectable in our assays. The p 40 subunit is not only shared by IL-12 and IL-23, but it also forms biologically active homodimers, capable of suppressing regulatory $\mathrm{T}_{\text {cells }}{ }^{22}$ or inducing $\mathrm{NO}^{23}$ and $\mathrm{TNF} \alpha^{24}$ production in microglia. It has been suggested that p 40 itself may play an important role in the pathogenesis of $\mathrm{MS}^{25}$ because of its strong proinflammatory activity and preferential mRNA expression in MS lesions. The clear inhibition of $\mathrm{p} 40$ production in the CSF indicates that daclizumab therapy suppresses intrathecal immune responses in MS.

Because some immune cells have important physiologic functions in the CNS, such as immunosurveillance, maintenance of adult neurogenesis, ${ }^{26,27}$ and promoting CNS reparative processes, ${ }^{28-30}$ the ability of daclizumab to induce effective immunoregulation without limiting access of the immune cells to the intrathecal compartment may have long-term advantage over therapies that cause indiscriminate depletion of the immune cells from the CNS. However, only large, properly controlled clinical trials and longitudinal studies will determine if daclizumab maintains long-term efficacy in MS without causing an increase in CNS infections or other side effects. Similarly, the effect of different disease-modifying therapies on endogenous repair processes in the CNS can only be derived from future carefully designed comparative studies.

\section{AUTHOR CONTRIBUTIONS}

Dr. Bielekova is coinventor on several NIH patents related to daclizumab and as such has received patent royalty payments from NIH. Dr. Richert is a full-time employee of and receives stock options in Biogen Idec; she became employee of Biogen Idec after study conclusion and after data analysis was finalized. M.L. Herman and J. Ohayon report no disclosures. Dr. Waldmann is coinventor on several NIH patents related to daclizumab and as such has received patent royalty payments from NIH. Dr. McFarland is coinventor on several NIH patents related to daclizumab and as such has received patent royalty payments from NIH. Dr. Martin has served on scientific advisory boards for Biogen Idec, Merck Serono, Teva Pharmaceutical Industries Ltd., and sanofi-aventis; has received speaker honoraria from Biogen Idec and Merck Serono; is coinventor on several NIH patents related to daclizumab and as such has received patent royalty payments from NIH; and serves as a consultant for the Myelin Repair Foundation, The Weatherall Institute for Molecular Studies, University of Oxford, and the Hertie Foundation. Dr. Blevins serves on scientific advisory boards and as a consultant for Biogen Idec, Teva
Pharmaceutical Industries Ltd., and Merck Serono; and has received funding for travel from the Cumming Foundation, Biogen Idec, Teva Pharmaceutical Industries Ltd., and Merck Serono.

\section{ACKNOWLEDGMENT}

The authors thank Azita Kashani, for technical assistance with data acquisition; Unsong Oh, MD, for patient care; Helen Griffith, RN, for nursing assistance and help with patient scheduling; nurses Angela Kokkinis, Diane Vollberg, and Jenifer Dwyer for nursing care; MRI technologists Ellen Condon, Sahra Omar, Renee Hill, and Jeanette Black for MRI data acquisition; and Roger Stone for maintaining the NIB database. Biostatistical analysis was performed by $\mathrm{B}$. Bielekova, MD, using predefined statistical measures and $p$ value adjustments for multiple comparisons according to the clinical trial protocol, which was reviewed and approved by NINDS Scientific Review Committee, which includes a professional biostatistician (employee of NIH/NINDS).

\section{DISCLOSURE}

B.B. designed immunological studies, supervised collection of immunological data, analyzed all data, and wrote the paper. N.R. supervised collection of MRI data, analyzed MRI data, and contributed to writing of the paper. M.H. performed detection of cytokines, including data analysis, and contributed to writing of the paper. J.O. collected clinical data and contributed to data analysis and writing of the paper. T.A.W. provided regulatory and intellectual contribution. H.M. and R.M. had supervisory role, provided intellectual contribution to study design, and together with B.B. were PIs during part of the study duration. G.B. designed clinical protocol, collected clinical data, supervised collection of immunological data, and was lead investigator on the trial.

Received March 16, 2011. Accepted in final form July 18, 2011.

\section{REFERENCES}

1. Bielekova B, Richert N, Howard T, et al. Humanized antiCD25 (daclizumab) inhibits disease activity in multiple sclerosis patients failing to respond to interferon-beta. Proc Natl Acad Sci USA 2004;101:8705-8708.

2. Bielekova B, Howard T, Packer AN, et al. Effect of antiCD25 antibody daclizumab in the inhibition of inflammation and stabilization of disease progression in multiple sclerosis. Arch Neurol 2009;66:483-489.

3. Rose JW, Burns JB, Bjorklund J, Klein J, Watt HE, Carlson NG. Daclizumab phase II trial in relapsing and remitting multiple sclerosis: MRI and clinical results. Neurology 2007;69:785-789.

4. Bielekova B, Catalfamo M, Reichert-Scrivner S, et al. Regulatory CD56bright natural killer cells mediate immunomodulatory effects of IL-2R-alpha-targeted therapy (daclizumab) in multiple sclerosis. PNAS 2006;103:59415946.

5. Wynn D, Kaufman M, Montalban X, et al. Daclizumab in active relapsing multiple sclerosis (CHOICE study): a phase 2, randomised, double-blind, placebo-controlled, add-on trial with interferon beta. Lancet Neurol 2010;9: 381-390.

6. Hao J, Liu R, Piao W, et al. Central nervous system (CNS)-resident natural killer cells suppress Th17 responses and CNS autoimmune pathology. J Exp Med 2010;207: 1907-1921.

7. Vandenbark AA, Huan J, Agotsch M, et al. Interferonbeta-1a treatment increases CD56bright natural killer cells and CD4+CD25+ Foxp3 expression in subjects with multiple sclerosis. J Neuroimmunol 2009;215:125-128.

8. McDonald WI, Compston A, Edan G, et al. Recommended diagnostic criteria for multiple sclerosis: guide- 
lines from the International Panel on the diagnosis of multiple sclerosis. Ann Neurol 2001;50:121-127.

9. Kurtzke JF. Rating neurologic impairment in multiple sclerosis: an expanded disability status scale (EDSS). Neurology 1983;33:1444-1452.

10. Schumacher GA. Problems of experimental trials of therapy in multiple sclerosis: Report by the panel on the evaluation of experimental trials in multiple sclerosis. Ann NY Acad Sci 1965;112:552-568.

11. Cutter GR, Baier ML, Rudick RA, et al. Development of a multiple sclerosis functional composite as a clinical trial outcome measure. Brain 1999;122:871-882.

12. Sharrack B, Hughes RA. Clinical scales for multiple sclerosis. J Neurol Sci 1996;135:1-9.

13. Richert ND, Howard T, Frank JA, et al. Relationship between inflammatory lesions and cerebral atrophy in multiple sclerosis. Neurology 2006;66:551-556.

14. Richert ND, Ostuni JL, Bash CN, Duyn JH, McFarland HF, Frank JA. Serial whole-brain magnetization transfer imaging in patients with relapsing-remitting multiple sclerosis at baseline and during treatment with interferon beta1b. AJNR Am J Neuroradiol 1998;19:1705-1713.

15. Ostuni JL, Levin RL, Frank JA, DeCarli C. Correspondence of closest gradient voxels-a robust registration algorithm. J Magn Reson Imaging 1997;7:410-415.

16. Muraro PA, Leist T, Bielekova B, McFarland HF. VLA-4/ $\mathrm{CD} 49 \mathrm{~d}$ downregulated on primed $\mathrm{T}$ lymphocytes during interferon-beta therapy in multiple sclerosis. J Neuroimmunol 2000;111:186-194.

17. Rose JW, Watt HE, White AT, Carlson NG. Treatment of multiple sclerosis with an anti-interleukin-2 receptor monoclonal antibody. Ann Neurol 2004;56:864-867.

18. Stuve O, Marra CM, Jerome KR, et al. Immune surveillance in multiple sclerosis patients treated with natalizumab. Ann Neurol 2006;59:743-747.

19. Stuve O, Marra CM, Bar-Or A, et al. Altered CD4+/ CD8 + T-cell ratios in cerebrospinal fluid of natalizumabtreated patients with multiple sclerosis. Arch Neurol 2006; 63:1383-1387.
20. Cross AH, Stark JL, Lauber J, Ramsbottom MJ, Lyons JA. Rituximab reduces $\mathrm{B}$ cells and $\mathrm{T}$ cells in cerebrospinal fluid of multiple sclerosis patients. J Neuroimmunol 2006;180: 63-70.

21. Rubenstein JL, Combs D, Rosenberg J, et al. Rituximab therapy for CNS lymphomas: targeting the leptomeningeal compartment. Blood 2003;101:466-468.

22. Brahmachari S, Pahan K. Suppression of regulatory T cells by IL-12p40 homodimer via nitric oxide. J Immunol 2009; 183:2045-2058.

23. Jana M, Dasgupta S, Pal U, Pahan K. IL-12 p40 homodimer, the so-called biologically inactive molecule, induces nitric oxide synthase in microglia via IL-12R beta 1 . Glia 2009;57:1553-1565.

24. Jana M, Pahan K. Induction of lymphotoxin-alpha by interleukin-12 p40 homodimer, the so-called biologically inactive molecule, but not IL-12 p70. Immunology 2009; 127:312-325.

25. Brahmachari $\mathrm{S}$, Pahan K. Role of cytokine $\mathrm{p} 40$ family in multiple sclerosis. Minerva Med 2008;99:105-118.

26. Ziv Y, Ron N, Butovsky O, et al. Immune cells contribute to the maintenance of neurogenesis and spatial learning abilities in adulthood. Nat Neurosci 2006;9:268-275.

27. Kipnis J, Cohen H, Cardon M, Ziv Y, Schwartz M. T cell deficiency leads to cognitive dysfunction: implications for therapeutic vaccination for schizophrenia and other psychiatric conditions. Proc Natl Acad Sci USA 2004;101: $8180-8185$.

28. Ziv Y, Avidan H, Pluchino S, Martino G, Schwartz M. Synergy between immune cells and adult neural stem/ progenitor cells promotes functional recovery from spinal cord injury. Proc Natl Acad Sci USA 2006;103:1317413179 .

29. Bieber AJ, Kerr S, Rodriguez M. Efficient central nervous system remyelination requires T cells. Ann Neurol 2003; 53:680-684.

30. Schwartz M, Kipnis J. Protective autoimmunity and neuroprotection in inflammatory and noninflammatory neurodegenerative diseases. J Neurol Sci 2005;233:163-166.

\section{Ramp Up Your Self-assessment with NeuroSAE ${ }^{\circledR}$}

We've ramped up the benefits without ramping up the price. That's right. In addition to meeting the self-assessment requirements for MOC, NeuroSAE IV now offers $6 \mathrm{CME}$ credits to help fulfill both the self-assessment and CME requirements of your $\mathrm{ABPN}$-required maintenance of certification.

- Assess your knowledge of neurology

- Compare your performance to other neurologists

- Use convenient online program

- Access from your mobile phone

Get started today at www.aan.com/view/NeuroSAE 